\title{
A systematic literature review of data forecast and internet of things on the e-health landscape
}

\author{
Gabriel Souto Fischer*, \\ Rodrigo da Rosa Righi, \\ Vinicius Facco Rodrigues and \\ Cristiano André da Costa
}

\author{
Academic Research and Graduate Unit, \\ Applied Computing Graduate Program, \\ Universidade do Vale do Rio dos Sinos, \\ São Leopoldo, Brazil \\ Email: gabrielr15xp@gmail.com \\ Email: rrrighi@unisinos.br \\ Email: vfrodrigues@unisinos.br \\ Email: cac@unisinos.br \\ *Corresponding author
}

\begin{abstract}
Internet of things (IoT) is a constantly expanding paradigm that promises to revolutionise healthcare applications and could be associated with several other techniques. Data forecast is another paradigm widely used, where data captured over time are analysed in order to identify and predict problematic situations that may happen in the future. After research, we did not find surveys that address IoT combined with data prediction in healthcare area in literature. In this context, this work presents a systematic literature review on internet of things applied to e-health landscape with a focus on data forecast, presenting as results 14 papers about this theme, and a comparative analysis between them. Our main contribution for literature is a taxonomy for IoT systems with data forecast applied to healthcare. Finally, this paper presents some possibilities and challenges of exploration in the study field, showing existing gaps for future approaches.
\end{abstract}

Keywords: internet of things; IoT; health; data forecast; sensors; distributed systems; taxonomy; healthcare environments; survey.

Reference to this paper should be made as follows: Fischer, G.S., da Rosa Righi, R., Rodrigues, V.F. and da Costa, C.A. (2019) 'A systematic literature review of data forecast and internet of things on the e-health landscape', Int. J. Computational Medicine and Healthcare, Vol. 1, No. 1, pp.34-58.

Biographical notes: Gabriel Souto Fischer received his BSC in Computer Science from the Universidade do Vale do Rio dos Sinos in 2016, and his MSc in Applied Computing from the Universidade do Vale do Rio dos Sinos in 2019. His research interests include internet of things, distributed applications, applications in healthcare, elasticity, and human resources management. 
Rodrigo da Rosa Righi received his BSc in Computer Science from the Universidade Federal de Santa Maria in 2003, his MSc and PhD in Computer Science from the Universidade Federal do Rio Grande do Sul in 2005 and 2009, respectively, and his Postdoctorate from the Korea Advanced Institute of Science and Technology in 2013. He is currently the Applied Computing Graduate Program coordinator and Research Professor in the Universidade do Vale do Rio dos Sinos. His research interests include internet of things, load balance and distributed applications.

Vinicius Facco Rodrigues received his BSc in Computer Science from the Universidade do Vale do Rio dos Sinos in 2012, and his MSc in Applied Computing from the Universidade do Vale do Rio dos Sinos in 2016. He is currently a $\mathrm{PhD}$ student in Applied Computing from the Universidade do Vale do Rio dos Sinos. His research interests include cloud computing and sensor networks.

Cristiano André da Costa received his BSc in Computer Science from the Universidade Católica de Pelotas in 1994, and his MSc and PhD in Computer Science from the Universidade Federal do Rio Grande do Sul in 1997 and 2008, respectively. $\mathrm{He}$ is currently an Applied Computing Graduate Program Research Professor in the Universidade do Vale do Rio dos Sinos. His research interests include ubiquitous and pervasive computing, mobile computing, context science, distributed systems, cloud computing, internet of things, high-performance processing, and applications in healthcare.

\section{Introduction}

Internet of things (IoT) is a computational concept where physical objects and 'things' are connected through a network structure and are part of the internet activities in order to exchange information about themselves and about objects and things around themselves (Singh and Kapoor, 2017). IoT is a concept of computing where everyday objects end up possessing some kind of hardware or embedded device that can connect to a network or simply be online. The development of this paradigm is in constant growth. This is because of the continuous efforts of the research community, because IoT allows unlimited applications to solve unlimited needs in all spheres of life. Thus, in a not-so-distant future, everything in our homes, workplaces and study will have a unique internet address and through the network it will be possible to monitor and control any of our 'things' (Singh and Kapoor, 2017). According to Sarhan (2018) IoT is a network of connected sensors, actuators, and everyday objects that are used in various domains, such airports, military and healthcare. IoT permits devices to interact not only with each other but with services and people on a global scale (Akeju et al., 2018). The world is undergoing an unprecedented technological transformation that evolves isolated systems to ubiquitous internet-enabled 'things' capable of generating and exchanging large amounts of valuable data. IoT is no longer just a concept but a new reality that is completely changing everyday life. In addition, it also promises to revolutionise healthcare applications, enabling a more personalised, preventive and collaborative way of caring for patients (Pinto et al., 2017).

According to Darshan and Anandakumar (2015), IoT remote health monitoring 
systems has various advantages over traditional health monitoring systems. Health sensors allows patients to wear them 24 hours by day for monitoring. A nurse or doctor can observe a patient for a limited time by day but critical health issues can occur at any moment. Based on this, 24/7 monitoring of health facts is crucial and it is necessary. In this context, IoT assisted patients can be accessed for medical staff over internet and by other systems, the health state of a patient can be supervised uninterruptedly, being possible to detect health risk situations at the right time, in order to being possible to apply the appropriate countermeasures. Also, IoT can support to collect health records that can be used to generate statistic information correlated to health condition. Using this statistics, surveillance and risk drawing of diseases can be completed using remote health data. Also according to Darshan and Anandakumar (2015), using IoT, systems are able to analyse and predict health disorders in early stage through data forecast techniques applied to generated data.

There are people who, when they have a health problem, can make an appointment in a clinic with a doctor they trust. Other people decide to go to the most appropriate hospital for their needs. In other cases, the health situation is so critical or emergency that the only alternative is to go as quickly as possible to hospital care. Thus, hospitals and healthcare environments are extremely important service points for the general population to perform one of the most important tasks: to ensure the maintenance of human life. There are many environments, resources and processes within a hospital to accomplish this task. Thus, it becomes interesting to have an efficient control of how these resources and processes are being used and executed in order to identify optimisation points. This becomes even more necessary when we come across the Brazilian reality, where hospitals are increasingly crowded with people to attend. When a hospital or emergency care unit is opened, usually it takes a long time until it exceeds its service capacity. If it were possible to identify when the points of care and resources would have a demand higher than their ability to attend, it would be possible to establish action plans to minimise or perhaps even eliminate these bottlenecks in the healthcare environments systems. However, how can we analyse and define when this moment is about to arrive? Relying only on the judgment of people who work with these resources, with no efficient way of recording their uses, and no statistically reliable forecast system can become a problem, with a high probability of errors in their evaluation.

Based on this, we can see that the use of data forecast combined with IoT applied to healthcare has several uses and a lot of potential, and can help to identify diseases and risk situations before they actually happen. After a literature review, we only find surveys that address IoTs and healthcare in a common context. When we extend our search for data forecast, we find few surveys that bring some question related to prediction techniques. This is the case of the review conducted by Darshan and Anandakumar (2015) on usage of IoT in healthcare system, and did not address the use of data forecast in any of reviewed articles. The relation of the research with prediction is in the results, since as the authors did not find an approach for prediction of chronic disorders in wearable devices context, they proposed a methodology for this. Another study is a survey conducted by Meharouech et al. (2015) on ubiquitous healthcare. The focus of the research was on the use of ubiquitous devices for the health area, and some of the several studies addressed, provided approaches for the mobility prediction of patients. Based on this, we can note that although there are surveys that address the 
use of IoT in the health area, there are still no surveys which combine data forecast in this area of study, that means there is no global view of it in the literature. Although there are taxonomies such as the one proposed by Meharouech et al. (2015), they focus only on IoT in the healthcare area and do not address data forecast.

In this context, in order to identify the state of the art for IoT systems with data forecast applied to healthcare and to discuss the main open issues, this work surveys the main contributions of the scientific community over the last years. Our purpose was to review the use of data forecast combined with IoTs in healthcare literature and describe the existing models. As a way of mapping this study scenario, we used the systematic literature review methodology to choose the studies (Roehrs et al., 2017). As a result, we reviewed scientific studies published in the last years, selected the most significant approaches, and exhaustively surveyed the use of IoT combined with data forecast in healthcare field. Also, we developed an updated and wide taxonomy for IoT systems with data forecast applied to healthcare and indicate challenges and possibilities for future study. We have also proposed contributions in our field from the study of related works, which are an updated taxonomy and an updated vision about main challenges and possibilities about the use of IoT with data forecast applied to healthcare.

This article is organised as follows: Section 2 presents the article selection process through a systematic literature review for Health IoT systems, bringing a comparison between the selected studies. Section 3 presents the state of the art for IoTs combined with data forecast in healthcare literature. This section also describes a taxonomy proposed in this work for IoT systems with data forecast applied to healthcare. Section 4 presents the possibilities and challenges for exploring the IoTs paradigm and data forecast techniques to improve healthcare processes and, finally, Section 5 presents the conclusions and proposed future works.

\section{Article selection}

In order to carry out a search for papers in an efficient and standardised way, the use of a research strategy known as a systematic literature review was defined, in order to have a process widely used in the academic community to carry out a survey of the different types of scientific work. Thus, this section presents a systematic review of the literature in order to identify and provide the state of the art of academic research in healthcare area, related to the concept of IoTs, focusing on data forecast to predict and prevent problems. This type of literature review was selected in order to summarise the technology in relation to this topic, in a way that does not require in-depth analysis and synthesis. For this systematic literature review, the following activities were used, as reviewed by Roehrs et al. (2017):

1 Research questions: Presents the research questions elaborated for this systematic review.

2 Search strategy: Describes the explored libraries and the strategy to collect the data.

3 Article selection: Define how the papers were selected, presenting the exclusion criterion used, as well as the steps taken to filter and select the studies. 
4 Distribution of studies: Present how selected studies are distributed chronologically.

5 Quality assessment: Describe the quality assessment of the selected studies.

6 Data extraction: Compare the selected papers and research questions.

\subsection{Research questions}

According to the studies of Roehrs et al. (2017), the definition of research questions is one of the most important parts of any systematic literature review. This study aims to identify and classify the technology related to IoT use in healthcare combined with data forecast; the resources, problems, challenges and solutions that are currently being considered; and the research opportunities that exist or are emerging. For this work, two types of questions were defined: the general question (GQ) and the specific questions (SQ). Table 1 presents all the research questions raised for this search of works related to the research theme of this document.

Table 1 Research questions

\begin{tabular}{|c|c|}
\hline Group and identifier & Question \\
\hline \multicolumn{2}{|l|}{ General question } \\
\hline GQ1 & $\begin{array}{l}\text { How would the taxonomy for IoT systems with data forecast applied to } \\
\text { healthcare appear? }\end{array}$ \\
\hline \multicolumn{2}{|l|}{ Specific questions } \\
\hline SQ1 & $\begin{array}{l}\text { What are the data that are included in an IoT system with data forecast } \\
\text { applied to healthcare? }\end{array}$ \\
\hline SQ2 & $\begin{array}{l}\text { What are the technologies and standards that apply to IoT systems with } \\
\text { data forecast applied to healthcare? }\end{array}$ \\
\hline SQ3 & $\begin{array}{l}\text { Which are the techniques or methods used to input information into IoT } \\
\text { systems with data forecast applied to healthcare? }\end{array}$ \\
\hline SQ4 & $\begin{array}{l}\text { What are the goals of IoT systems with data forecast applied to } \\
\text { healthcare? }\end{array}$ \\
\hline SQ5 & $\begin{array}{l}\text { What are the models of architecture of IoT systems with data forecast } \\
\text { applied to healthcare? }\end{array}$ \\
\hline
\end{tabular}

The proposed GQ brings a broad classification related to the IoT systems with data forecast for the healthcare area. GQ1 refers to the question of classifying and defining a taxonomy for existing IoT systems with data forecast applied to healthcare. To improve the filtering process of the studies, in order to guide and facilitate the process of identifying the answer for GQ1, some SQ were defined. SQ1 seeks to identify the types of data a IoT system with data forecast applied to healthcare can contain. SQ2 investigates the types of standards that are used for IoT systems with data forecast applied to healthcare. SQ3 seeks to show how information is inserted into the proposed systems. SQ4 investigates what are the goals that IoT systems with data forecast applied to healthcare have. SQ5 focuses on architecture models for IoT systems with data forecast applied to healthcare. 


\subsection{Search strategy}

The next step was to identify a set of studies related to research questions. In this process, the search keywords and the definition of the search scope were defined. These words were defined in order to obtain research results focused on the theme of this systematic review. Therefore, the search string located in Figure 1 was defined for the search and selection of articles in electronic academic databases.

Figure 1 Search string

((prediction OR analytics OR forecast OR time serie) AND (Internet of Things OR IoT OR sensors) AND (health OR healthcare OR hospitalar OR clinical))

In order to cover a large number of related studies, several electronic scientific libraries were selected as the basis for research on the articles, listed in Table 2. According to Roehrs et al. (2017), these portals are some of cover the most relevant journals and conferences of the computer science area.

Table 2 Electronic databases for article search

\begin{tabular}{lc}
\hline Acronym & Electronic database name \\
\hline ACM & ACM Digital Library \\
IEEE & IEEE Xplore Digital Library \\
IET & IET Digital Library \\
PubMed & National Center for Biotechnology Information, US National Library of \\
& Medicine \\
ScienceDirect & Elsevier B.V. ScienceDirect \\
Wiley & Wiley Online Library \\
\hline
\end{tabular}

\subsection{Article selection}

After all articles were found in the electronic databases using the search string, we carried out a process of removing articles that would not be relevant to this research, in order to keep only those that would be more representative to the theme. Thus, all works that did not address the use of IoTs combined with data forecast to prevent problems in healthcare were removed. Therefore, the following exclusion criterion were defined for article selection:

- Exclusion criterion 1: Article does not address the use of IoTs in healthcare.

- Exclusion criterion 2: Article does not address data forecast techniques to prevent problems.

According to Roehrs et al. (2017), an important step in the systematic review is the definition of the filtering process of the articles. As a result, the following steps were defined for the filtering and selection process: 
1 filter by title

2 filter by abstract

3 removal of duplicates e

4 filter by full text.

First, the titles of the articles were analysed, so that those who did not address the IoTs and health were excluded, according to exclusion criterion 1. After, the abstract of the remaining articles were analysed so that the articles that do not address the use of IoT in healthcare could be excluded, according to exclusion criterion 1. At the same stage, we also excluded the articles in which nothing was found in their abstracts regarding data forecast, according to exclusion criterion 2. The remaining articles were then grouped and a search was conducted for duplicate jobs, according to the same study sometimes be available in more than one electronic library, and them were deleted. Finally, the texts of the articles filtered in the previous stages were read and the exclusion criterion were applied again, removing those that did not address the proposal of that systematic review.

\subsection{Quality assessment}

According to Roehrs et al. (2017), it is essential to evaluate the quality of the selected papers, in order to verify if it is really relevant to study. Thus, the selected articles were evaluated in relation to the parameters used by Roehrs et al. (2017): purpose of the research, contextualisation, literature review, related works, methodology, results obtained and the conclusion, related to the proposed objectives, and indication of future works. Based in Table 3 was elaborated, presenting the quality criteria in the form of questions to which the selected articles were submitted for validation of their quality.

Table 3 Quality assessment criteria

\begin{tabular}{ll}
\hline Identifier & \multicolumn{1}{c}{ Criteria } \\
\hline $\mathrm{C} 1$ & Does the article clearly show the purpose of the research? \\
$\mathrm{C} 2$ & $\begin{array}{l}\text { Does the article adequately describe the research context through a literature } \\
\text { review? }\end{array}$ \\
$\mathrm{C} 3$ & Does the article present the related work to the main contribution? \\
$\mathrm{C} 4$ & $\begin{array}{l}\text { Does the article have a description of a proposed architecture or research } \\
\text { methodology? }\end{array}$ \\
$\mathrm{C} 5$ & $\begin{array}{l}\text { Does the article present results for the research? } \\
\mathrm{C} 6\end{array}$ \\
$\mathrm{C} 7$ & Does the article present a study conclusion related to the research objectives? \\
\hline
\end{tabular}

\subsection{Data extraction}

In order to gather the information about the paper previously raised, a comparison was made between the chosen articles and the research questions. Table 4 shows how we 
did this comparison, bringing each item of the related papers to the research, in order to have a better way to extract details of the articles and understand how studies have addressed issues related to the research questions proposed in the systematic literature review performed in this paper.

Table 4 Review of articles with research questions

\begin{tabular}{lcc}
\hline Section & Description & Research questions \\
\hline $\begin{array}{l}\text { Metadata } \\
\text { Title }\end{array}$ & Title of article & \\
Abstract & Summary of paper with method and results & GQ1, SQ1, SQ2, SQ5 \\
Keywords & Words representing the article content & GQ1, SQ1, SQ2, SQ5 \\
$\begin{array}{l}\text { Article } \\
\text { Introduction }\end{array}$ & Section introducing the adressed problem & All questions \\
Background & Section presenting concepts about the article theme & All questions \\
Method & Presents the proposed scientific methodology & All questions \\
Results & Covers results acquired after evaluation & All questions \\
Discussion & Compare article results with the literature & SQ2-SQ5 \\
Conclusion & Relate objectives and hypotheses & SQ2-SQ5 \\
\hline
\end{tabular}

\subsection{Article selection process}

In the selection process, 9,486 articles were found in the initial search in the electronic libraries, before any exclusion or filtering criterion were applied, only using the search string. Figure 2 shows the entire filtering process used on these articles to search for related papers.

Figure 2 Systematic review filtering process (see online version for colours)

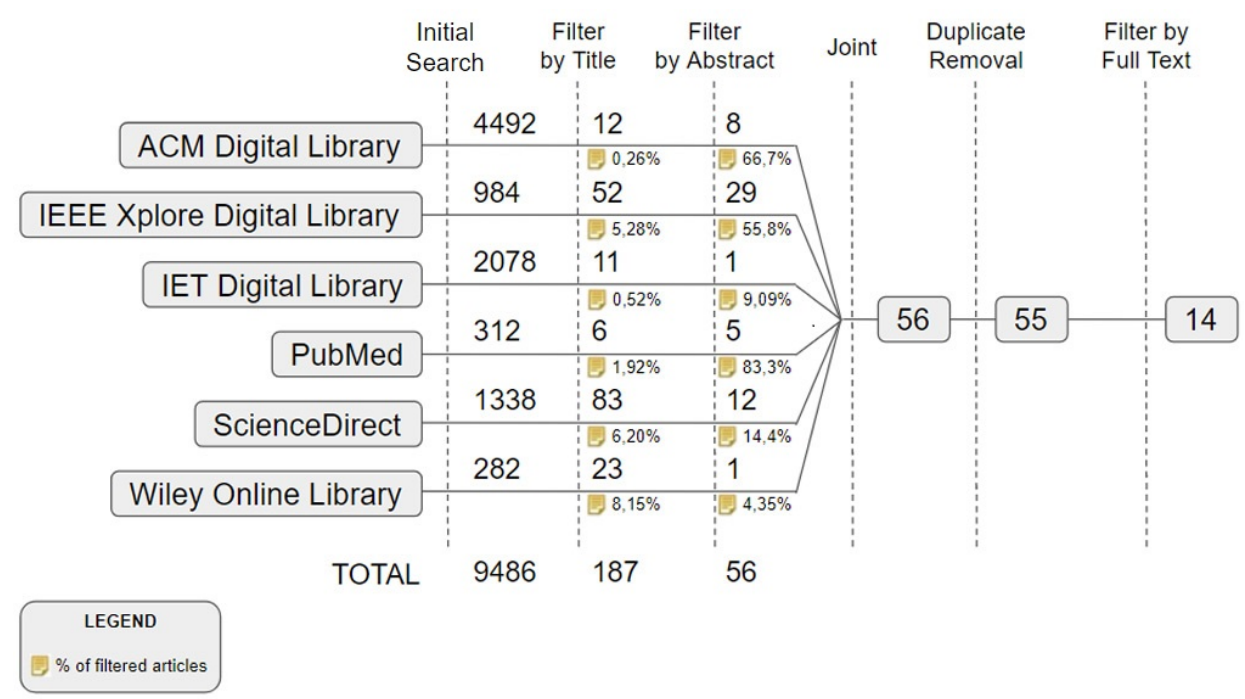


First, a filter by title process was carried out, based on exclusion criterion 1 . In this step, all articles that did not contain IoTs and health in their titles (using different words that could represent the same concepts in the literature) were eliminated. For this purpose, we used the refinement mechanisms of the electronic libraries. However, not all of them were able to perform an adequate refinement, being necessary the use of other techniques to carry out this process in an automated way. This was the case of the database ACM Digital Library, where the filters generated several impurities, since they could not differentiate the title of the article from titles of the conferences or journals. In order to perform this impurities removal, we used the program EndNote X8, a bibliographic management software for the publication of scientific articles (EndNote, 2017). Afterwards, the remaining titles were manually reviewed, eliminating those who used the IoT for 'health' of objects or services and not people. After this filtering process by title, 9,299 (98.03\%) works were eliminated, remaining 187 (1.97\%) articles. This occurred because the libraries search tool such as ACM and IET generated many impurities, blending articles that had mention to IoT and healthcare with several diverse studies published in conferences on health or IoT, for example.

Continuing the process, the filter by abstract of the works was carried out, using this time as exclusion criterions 1 and 2 . In this new stage, all articles that did not contain forecast techniques in their abstract (using different words that could represent the same concepts) were eliminated. Afterwards, the remaining works were revised manually, eliminating all those who did not present any IoTs use for health or nothing regarding data forecast in their abstracts. After this filter by abstract process, 131 (70.05\%) works were eliminated, remaining $56(29.95 \%)$ articles. The remaining works were grouped and $1 / 56(1.78 \%)$ were identified as duplicates and were eliminated, with $55(98.22 \%)$ remaining.

Finally, filter by full text was performed, where both defined exclusion criterion were used again. For this final step, the entire process was done manually, where all articles were read in whole or in part, and those that did not address the research theme proposed for this survey were eliminated. Thus, 42/56 (75\%) articles were excluded. The articles excluded in this step, for the most part, cite others studies that use data forecast, without proposing or using any technique in that context. Finally, the remaining 14 papers from all the processes were selected as results for the systematic literature review proposed in this research.

\subsection{Selected studies}

In Table 5 all previously selected articles are presented, bringing:

1 an identifier for each of them

2 author names in bibliographic reference format, along with the year of publication

3 the article publisher, e

4 type of publication, which may be journal article or conference article.

The papers were sorted in ascending order by year of publication and, after, order by alphabetical order of authors' names.

Figure 3 presents the chronological distribution of the selected works over the years, between 2010 and 2018. In the figure, each publication is represented by a block 
that displays your identifier and publisher inside, and the type of the article can be distinguished through the format of the block, as described in the legend. Above each set of publications, the number of articles published that year is displayed.

Table 5 Selected articles

\begin{tabular}{lccc}
\hline Identifier & Authors (year) & Publisher & Type \\
\hline A01 & Kwon et al. (2010) & IEEE & Conference \\
A02 & Spanakis et al. (2014) & IEEE & Conference \\
A03 & Vargheese and Viniotis (2014) & IEEE & Conference \\
A04 & Kan et al. (2015) & IEEE & Conference \\
A05 & Kang et al. (2015) & IEEE & Conference \\
A06 & Orimaye et al. (2015) & IEEE & Conference \\
A07 & Jouini et al. (2016) & IEEE & Conference \\
A08 & Azimi et al. (2017) & ACM & Journal \\
A09 & Chen et al. (2017) & Elsevier & Journal \\
A10 & Robben et al. (2017) & IEEE & Journal \\
A11 & Zamanifar et al. (2017) & Elsevier & Journal \\
A12 & Ali et al. (2018) & Elsevier & Journal \\
A13 & Farahani et al. (2018) & Elsevier & Journal \\
A14 & Rahmani et al. (2018) & Elsevier & Journal \\
\hline
\end{tabular}

Figure 3 Chronological distribution of studies (see online version for colours)

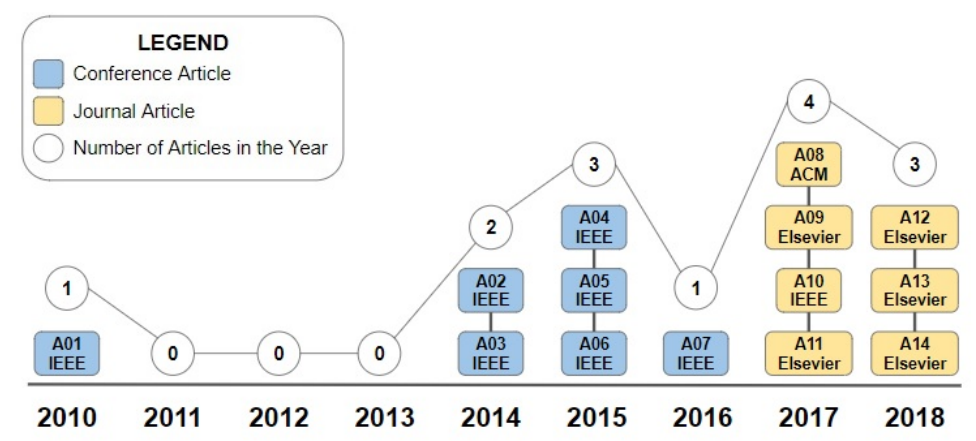

\subsection{Performing the quality assessment}

In Figure 4 the satisfied quality criteria are presented for each of the selected works, based on the quality assessment criteria proposed in Table 3 . The criteria satisfied by each article are shown on the vertical axis, and the articles themselves on the horizontal axis, according to the identifiers previously defined. After this quality assessment, it may be noted that most of the articles could reach six or seven quality criteria. Of those who have met only six criteria, most of them do not comment or cite any future work, because they are conclusive articles, that is, all their proposed objectives have been 
fulfilled. The article that has only five satisfied criteria, does not bring related work and does not present results because it is only an architectural proposal, without having been effectively implemented, leaving this implementation for a future work.

Figure 4 Satisfied quality criteria for each selected article (see online version for colours)

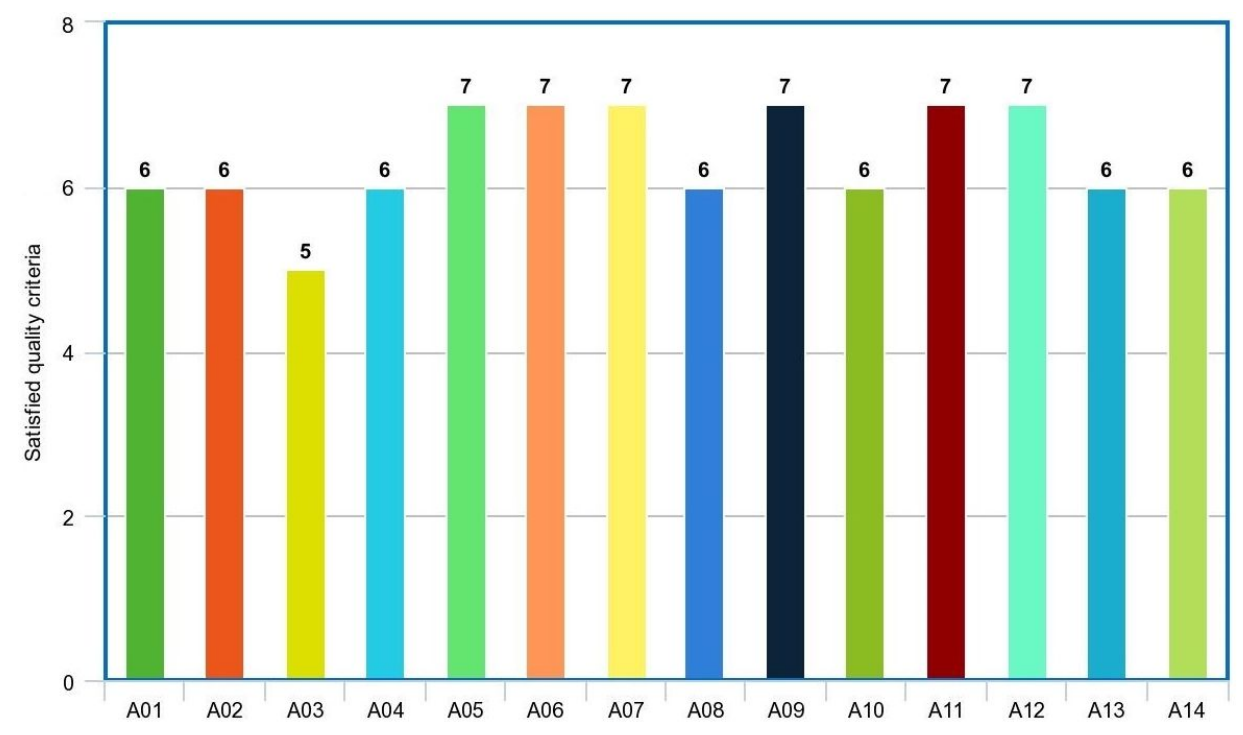

\section{State of the art}

Based on the selected articles, we can identify that there are currently several fronts and approaches when the objective is to predict issues related to the health of a patient using the IoTs and data forecast concepts. Through the articles surveyed it was possible to see that it is not only possible to use the technology for this, as it is already being used in several approaches in the scientific community. Most of the IoT systems with data forecast applied to healthcare researched focus on the monitoring of the patient's health conditions, in order to generate alerts if any risk situations are identified. These systems are able to predict when the patient's vital signs will be at risk, identify heart problems, treatment efficacy and environmental risk situations for patients. Table 6 presents a comparison between the collected papers, relating some of their main characteristics, showing some gaps in each one of the approaches.

The first group of characteristics presents mechanisms for security such as:

1 authentication and authorisation of users

2 data encryption

3 patient privacy.

The second group of characteristics shows which systems use:

4 cloud computing 
5 fog computing

6 data forecast.

With respect to data forecast, the following groups of characteristics serve to: show the techniques to predict, through,

7 artificial intelligence (AI)

8 big data

9 probabilistic and statistics approaches.

Show the AI techniques to predict, through,

10 random forest analysis

11 artificial neural networks (ANN)

12 fuzzy logic.

Show the probability and statistics models to predict, through,

13 disease combination appearance probability (DCAP) prediction algorithm

14 Gaussian function model

15 Bayesian inference model

16 Support vector machines (SVM) learning model

17 regression models

18 hidden Markov model

19 grey model.

And they also serve to show what predictions the systems are capable of making, such as:

20 the future status of vital signs and conditions of the patient's health

21 the incidence of heart disease

22 check if diseases are evolving

23 predict whether the treatments will be effective

24 anticipate risk situations for patients

25 identify the patient's future location for non-invasive sensors to act on it as it moves.

Based on the research questions defined in Subsection 2.1, we performed the data extraction process of the selected articles in order to determine the state of the art of IoTs and data forecast in healthcare systems. Next, we have the GQ and the SQ used to help answer the GQ, along with the answers found for each of them. 
Table 6 Comparative between characteristics of the articles

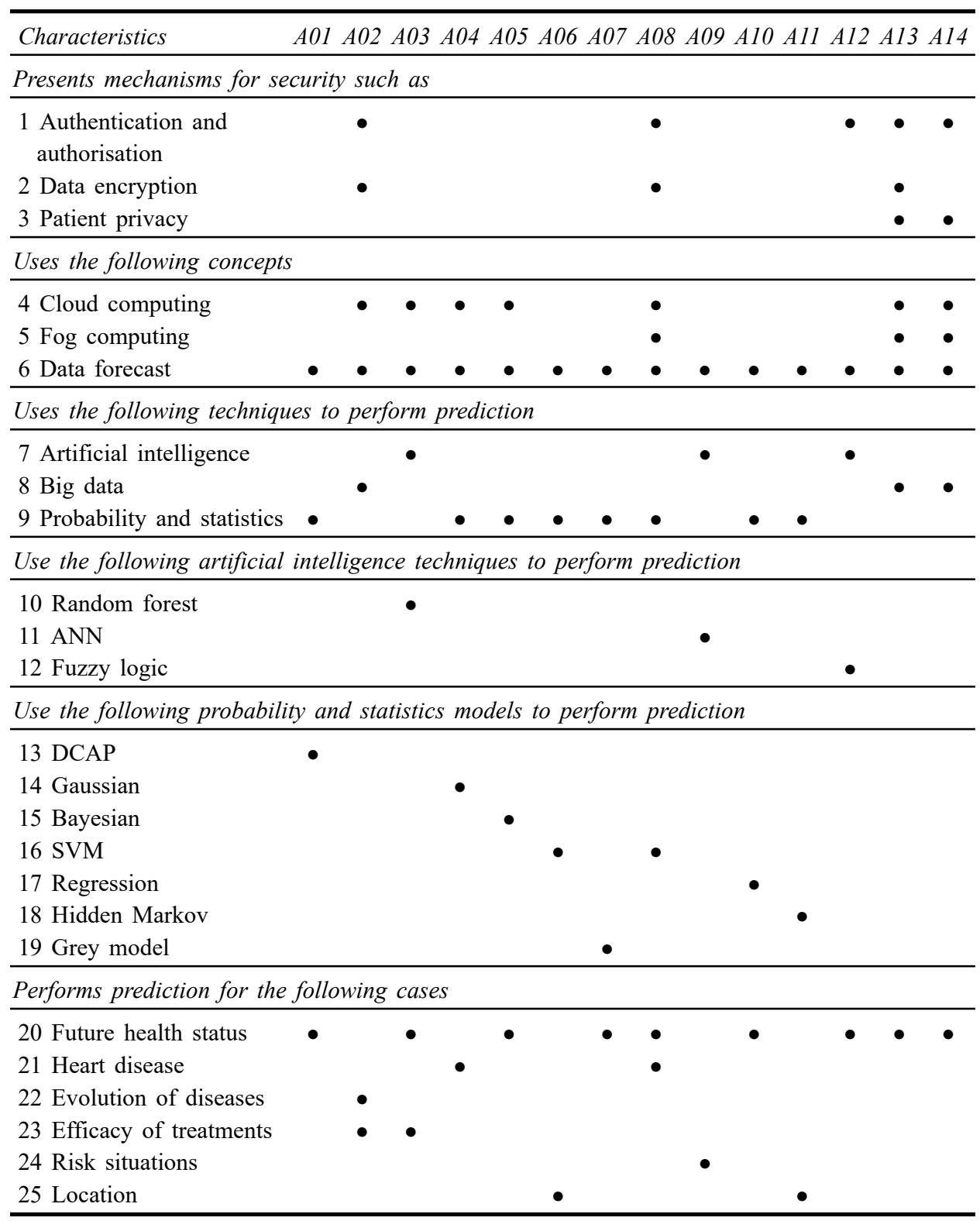

3.1 GQ1: how would the taxonomy for IoT systems with data forecast applied to healthcare appear?

We investigated several studies that work with the concept of IoTs and data forecast applied to situations in healthcare area. In this way, it was possible to construct a taxonomy to gather and organise the various possibilities of IoT systems with data forecast applied to healthcare. When analysing the selected articles, a taxonomy was proposed based on important characteristics of the models, in order to help in the 
classification, comparison and evaluation of different IoT systems for health. In addition, this classification can provide an overview of possible alternatives in terms of objectives, content and architectures. The proposed taxonomy is summarised in Table 7, divided into three groups:

1 structures

2 functions

3 architecture.

Next to each item have a brief description.

Figure 5 IoT systems with data forecast applied to healthcare taxonomy (see online version for colours)

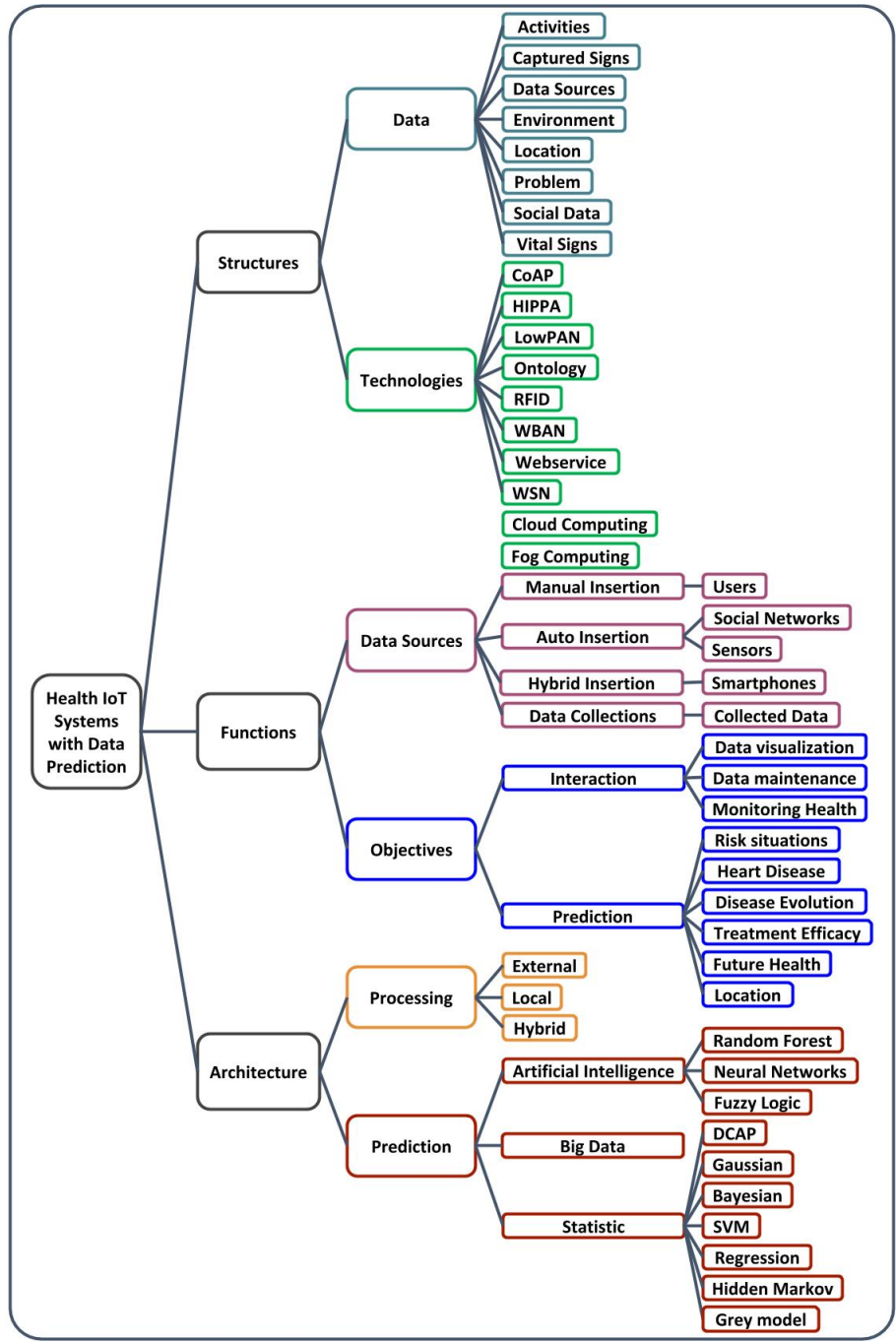


Table 7 IoT systems with data forecast applied to healthcare taxonomy summarised

\begin{tabular}{lc}
\hline Groups & Description \\
\hline $\begin{array}{l}\text { Structures } \\
\text { Data }\end{array}$ & $\begin{array}{r}\text { Data and technologies used in IoT systems with data forecast } \\
\text { Dechnologies }\end{array}$ \\
$\begin{array}{l}\text { Functions } \\
\text { Data source }\end{array}$ & $\begin{array}{c}\text { Technologies that can be applied in the systems (see Subsection 3.3) } \\
\text { Objectives }\end{array}$ \\
$\begin{array}{l}\text { Techniques for input information in the systems (see Subsection 3.4) } \\
\text { Architecture }\end{array}$ & Represents the aim of the systems (see Subsection 3.5) \\
Models & Architecture types (see Subsection 3.6) \\
Prediction & Describes the architecture models \\
\hline
\end{tabular}

Figure 5 presents our proposed taxonomy, bringing our entire classification for IoT systems with data forecast applied to healthcare. The next sections of this article present the subclassifications proposed in Table 7, showing how our taxonomy was proposed, also presenting which studies have used each of the structures, functions and architectures proposed in this work.

\subsection{SQ1: what are the data that are included in an IoT system with data forecast applied to healthcare?}

To answer this question, we have analysed all previously selected articles. The types of data that are included in an IoT systems with data forecast applied to healthcare are described in Table 8. The data types found are as follows:

1 activities that a particular patient may be performing

2 status of the environment where the patient is located

3 data sources, internal or external, with information on diseases, treatments, diagnoses and others

4 data captured through social networks

5 location of the patient in an environment in any instant of time

6 patient's vital signs captured by the system in real-time

7 patient's vital signs captured by examinations and measurements, subsequently inputted into the system.

Table 8 lists all data types found in the articles and a brief description of each of them.

\subsection{SQ2: what are the technologies and standards that apply to IoT systems with data forecast applied to healthcare?}

Many studies use own formats to organise the data they use, so that there is not always a standard among them. Thus, there are many technologies that can be used 
for IoT systems with data forecast applied to healthcare. However, in order to answer this question, we analysed the open industry standards and other technologies that are used in these articles. Table 9 presents them, some of them being: constrained application protocol (CoAP), low-power wireless personal area networks (LoWPAN), radio frequency identification (RFID), wireless body area network (WBAN) and wireless sensor network (WSN).

Table 8 Data found in IoT systems with data forecast applied to healthcare

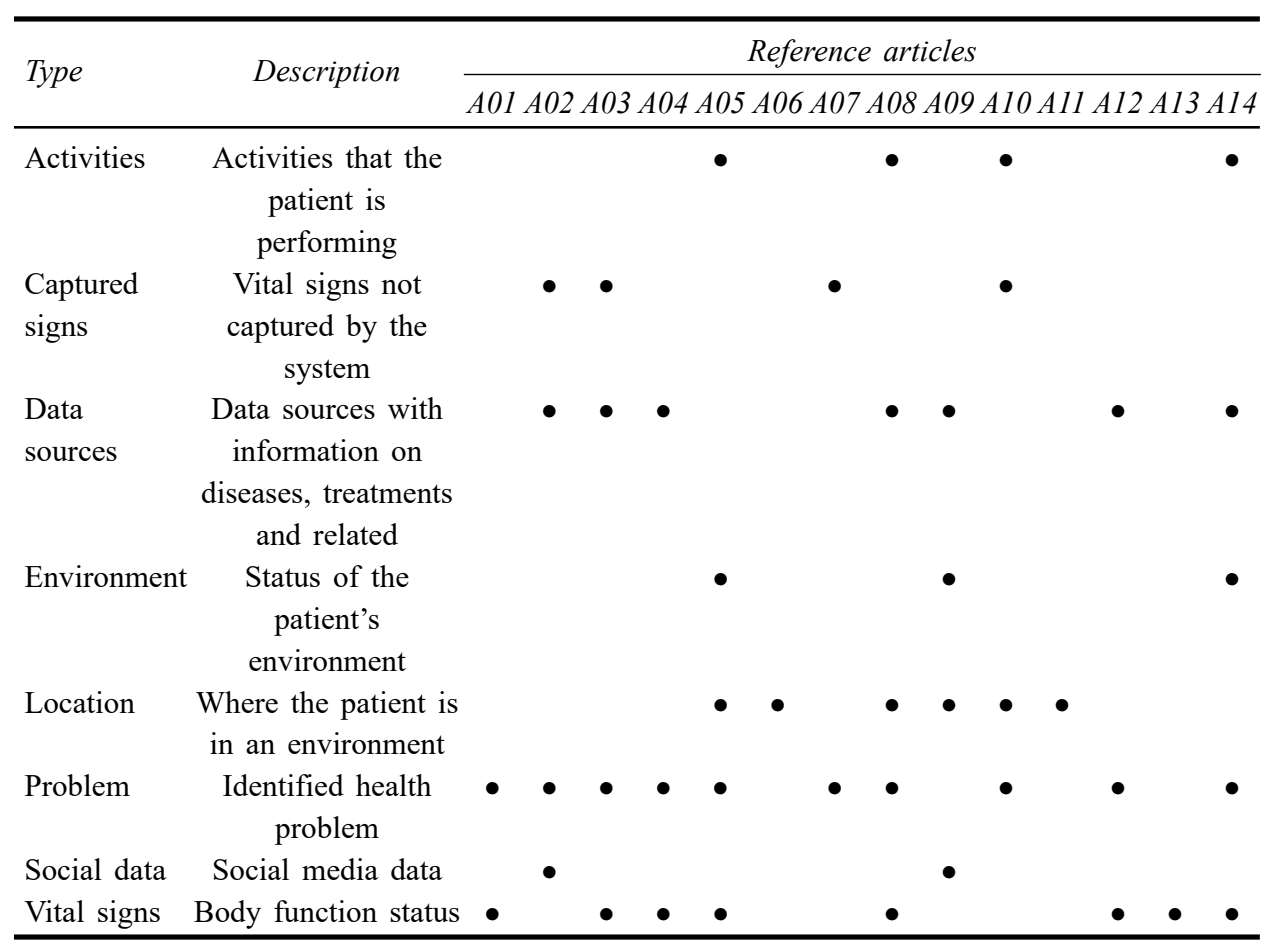

Table 9 IoT systems with data forecast applied to healthcare related technologies

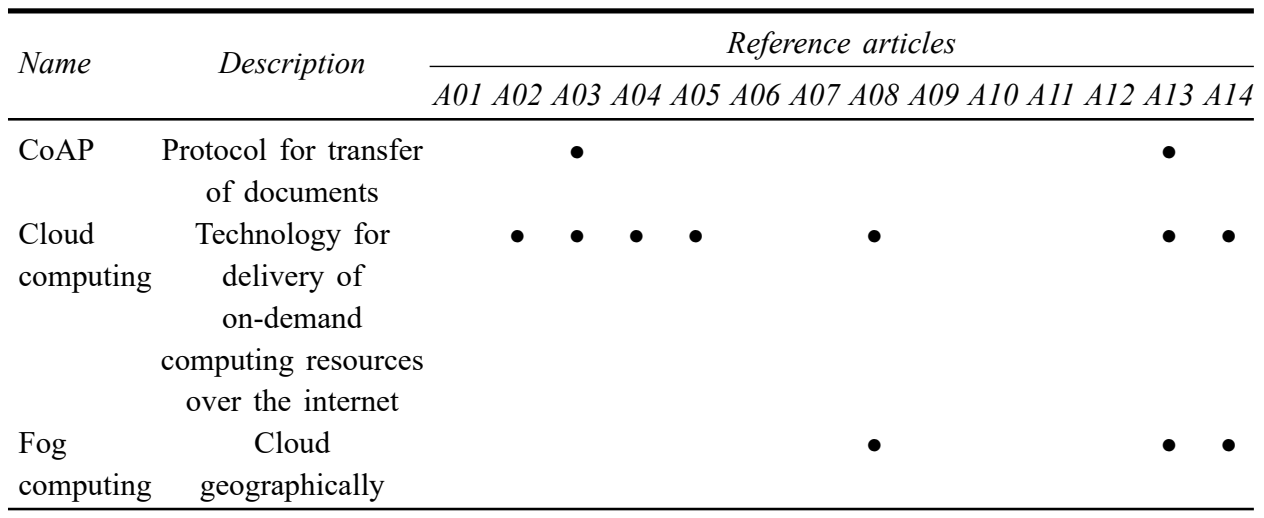


Table 9 IoT systems with data forecast applied to healthcare related technologies (continued)

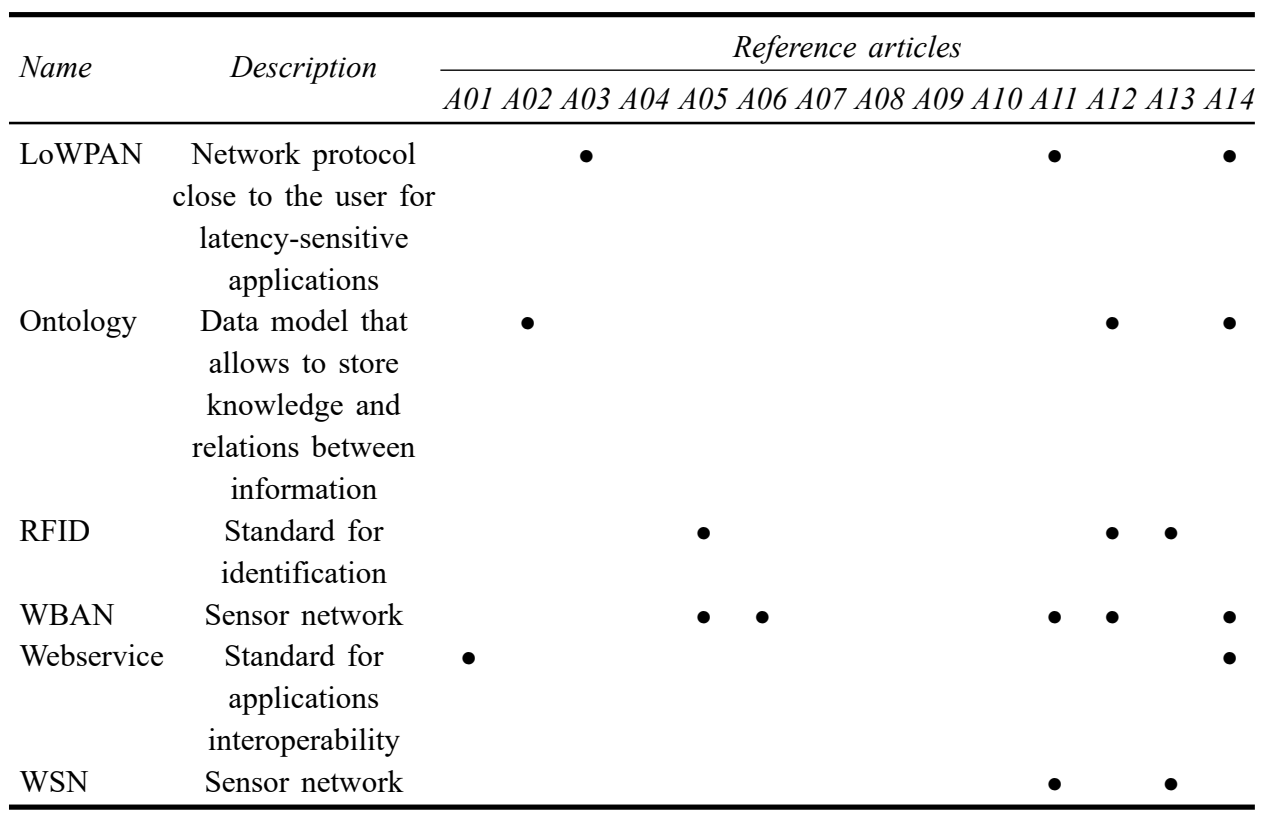

3.4 SQ3: which are the techniques or methods used to input information into IoT systems with data forecast applied to healthcare?

Another result of this systematic literature review was the identification of the techniques used by the IoT systems with data forecast applied to healthcare to perform the insertion of data and information into the applications. Table 10 presents the techniques for insertion of the data in the systems proposed in these articles, bringing a description of each of these techniques. The techniques are divided into four main groups: data collection (T1), where the data is inserted through previously created collections of data; auto insertion (T2), where information is captured automatically through some techniques and entered into the application; manual insertion (T3), where information must be entered manually into the application; hybrid insertion (T4), where the same technique can enter information in the system either manually or automatically.

\subsection{SQ4: what are the goals of IoT systems with data forecast applied to healthcare?}

This research question presents the main goals or objectives of IoT systems with data forecast applied to healthcare. Table 11 presents the objectives found for the articles analysed in this systematic literature review. We conclude that the objectives can be divided into two categories: interaction $(\mathrm{O} 1)$, that are related to the way of the system will interact with the patients and others users, and prediction $(\mathrm{O} 2)$, which are related to the predictions that the system intends to perform in order to prevent in advance life-threatening situations which patients may be exposed. 
Table 10 Techniques used to input information into IoT systems with data forecast applied to healthcare

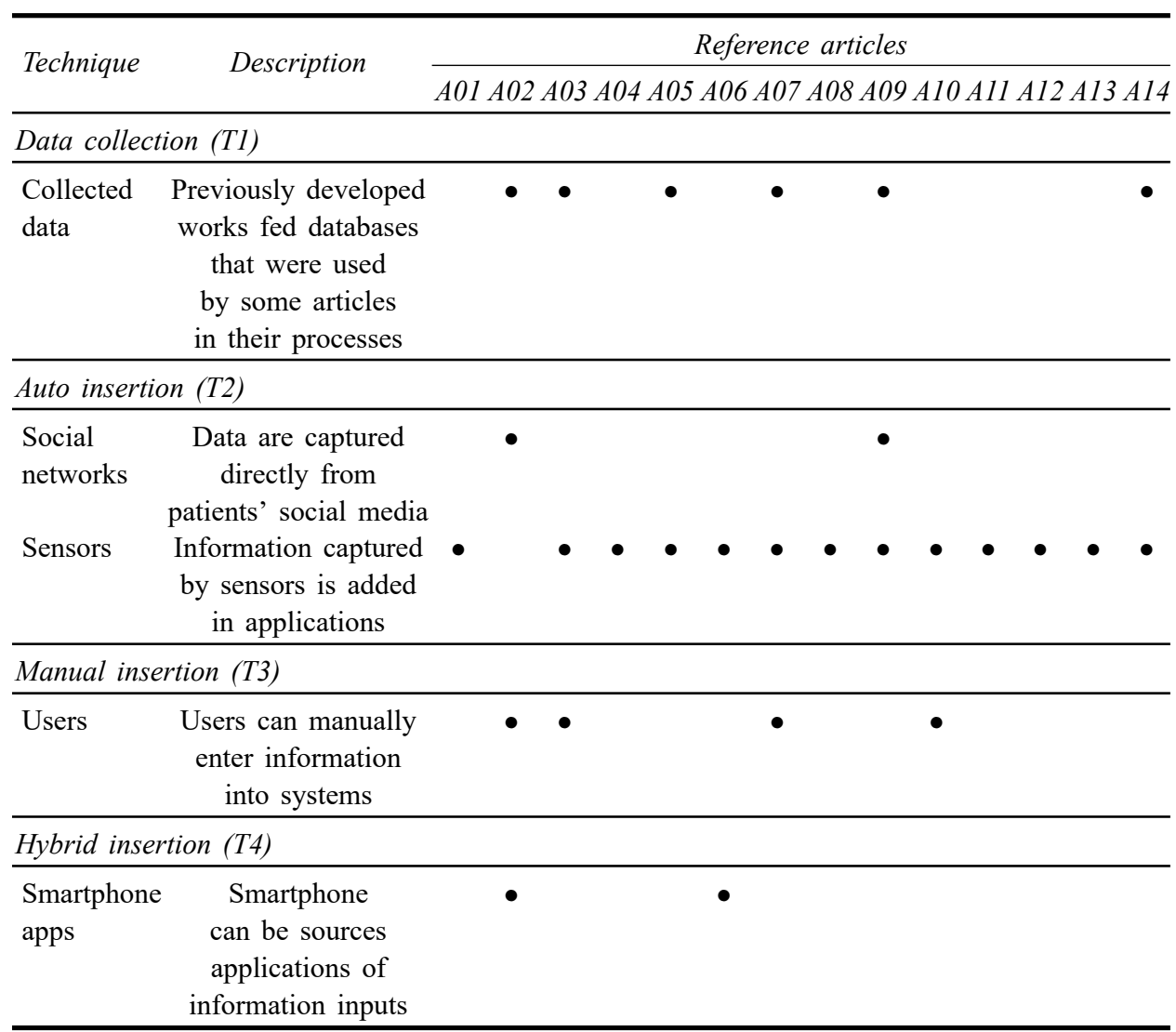

Table 11 Objectives related to the form of interaction with the system and to the predictions that the system intends to perform

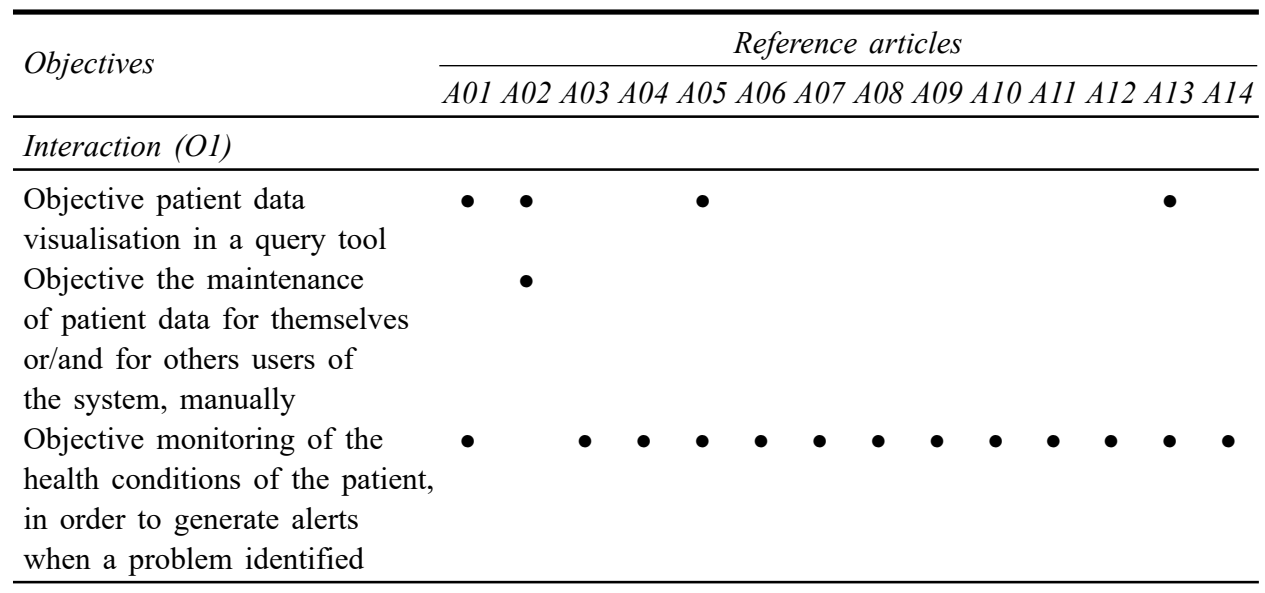


Table 11 Objectives related to the form of interaction with the system and to the predictions that the system intends to perform (continued)

\begin{tabular}{l}
\hline Objectives \\
\hline Prediction (O2) \\
\cline { 2 - 3 } Predict risk situations \\
that patients may be exposed \\
Predict heart disease \\
Predict disease evolution \\
Predict treatment efficacy \\
Predict future conditions \\
or vital signs of patients' health \\
Predict where the patient \\
will be next, in order to prepare \\
the sensors installed in the \\
healthcare environment to \\
collect the patient information \\
in a non-invasive way
\end{tabular}

Table 12 Models of data processing and prediction

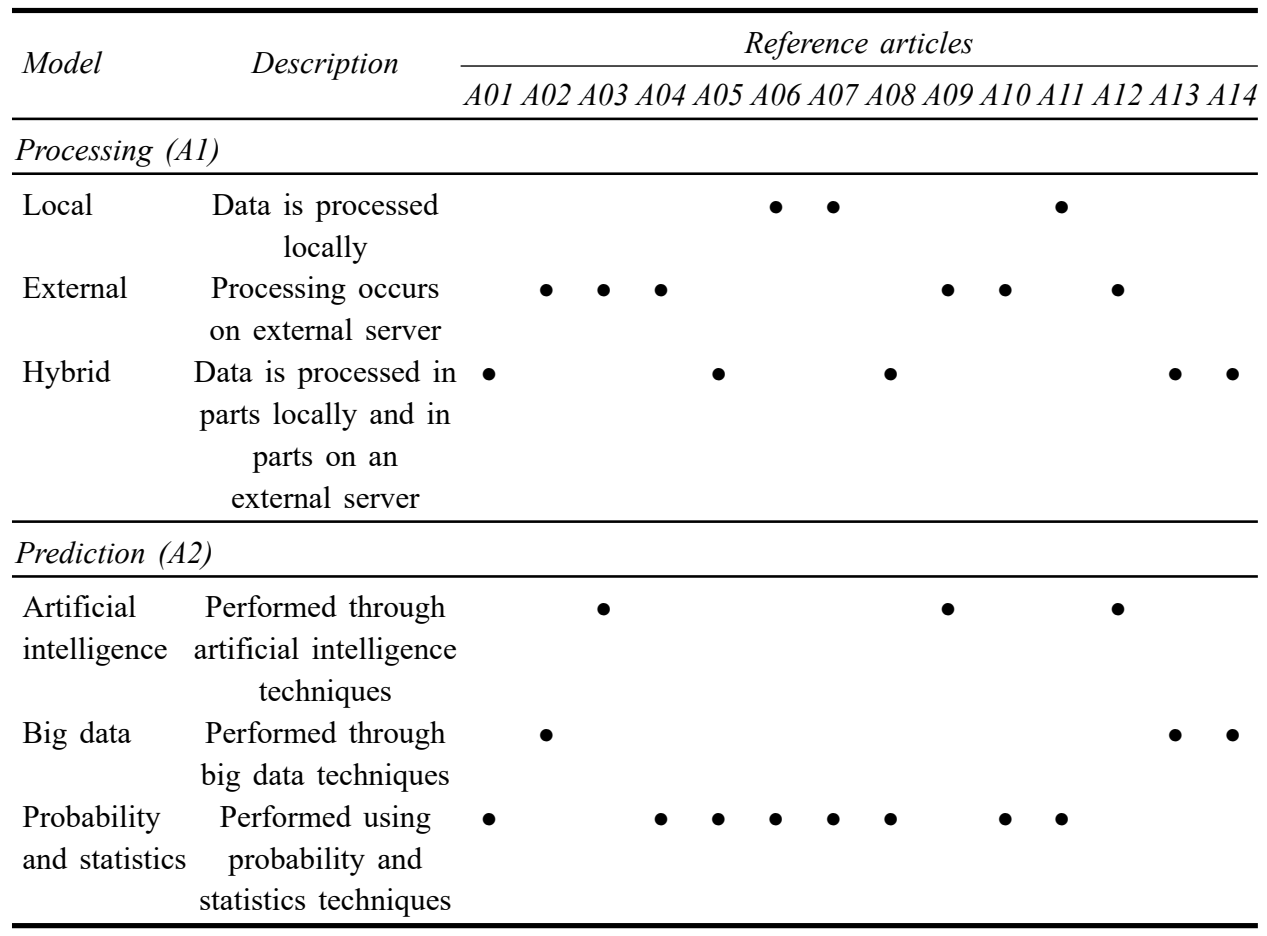




\subsection{SQ5: what are the models of architecture of IoT systems with data forecast applied to healthcare?}

This last specific question aims to identify the main models of architectures used by IoT systems with data forecast applied to healthcare in their implementations. After analysing the articles, we define two main architecture groups (as shown in Table 12): processing (A1), which presents the data processing architectures, which are classified in local, external or hybrid, and prediction (A2), which presents the techniques used for the data forecast architecture, such AI, big data, and probability and statistics.

\section{Challenges and possibilities}

We can observe that there are currently several fronts and approaches when the objective is to take care of the health of patients using the IoTs concept, and not only this, but to anticipate problem situations through data forecast techniques. Several systems use different techniques to achieve the same intentions. When we look at these systems, is possible to note that concepts such as cloud computing, fog computing, AI, big data, probability, and statistics are at the top of the world's health research. However, few of these approaches have effective mechanisms to deal with the issue of security in the digital age. And those who have them do not always rely on extremely secure protocols and systems. Some applications do not make use of adaptive computing resources like cloud computing, using own resources and own servers that end up becoming single points of failure and, because of that dependability, if a single service stops working, the entire application will fail.

Another point that can be seen is that there are many techniques for analysing the future status of the patient's health conditions, but practically none to predict the evolution of the diseases that the patient already has. The approaches are very focused on analysing patient health conditions, and focus very little on risk situations in the environment where the patient is inserted. Even in this regard, neither approach attempts to analyse the use of hospital resources and, consequently, to predict when these resources will become insufficient to care for the patients. Thus, we can say that the technology needed to better explore the IoTs concept and data forecast in healthcare Environments exists and is already used today, but there are few approaches that can merge several concepts into one to solve several of the problems raised.

\subsection{Distributed environments and dependability}

We can note that only half of the approaches studied make use of cloud computing and the other half operate on own, apparently centralised, servers. One of the main problems of working with a centralised environment is dependability, that is, with the system running on a single server, if it fails, the entire application will fail. Often, there are several services and servers to perform all the tasks, which makes the problem even worse, because if any one of them fails, the whole application stops working, and we will have several services and servers running, but nothing to process.

In this context, a possibility of exploration would be to create techniques and systems capable of operating over distributed environments, with data replication and redundancy of services and servers. One way to do this would be by using the cloud 
computing concept, maintaining the health IoT application with data forecast in a cloud instance. However, one of the major problems of IoT is the large amount of data generated by the most diverse sensors. This way, transmitting all the data to the cloud can take considerable time, and until the data is processed by the application, the problem that is waiting to be predicted will already have happened. Thus, another possibility of exploration would be to propose systems that use fog computing, that is, clouds closer to the user, making the transmission occur faster and, in this context, to create techniques to pre-process the data in an appropriate way, eliminating noise and sending only what is needed for the main cloud to perform data processing.

Also in this context of distributed applications, another possibility of exploration would be to define techniques for virtualisation of services, that is, to define techniques to instantiate virtual machines to meet the processing demand, and to perform load balancing between them. Thus the system would become able to increase and decrease the capacity as required. In this possibility, ways could be proposed to identify when a system is close to reaching its processing limit, to trigger a new virtual machine and, at the same time, to identify the underutilisation of the system to disable virtual machines. Also in this context, techniques could be proposed to divide the tasks between the machines appropriately.

\subsection{IoTs standards and technologies}

One of the major problems and challenges for the IoTs is the absence of standards for data. The proposed systems have different ways of storing data, performing processing, and making it available to users. Data captured by one application can hardly be used by another. The few data that can be used, need an intermediate processing, only to carry out the translation of the same between the applications. Some approaches used the CoAP for data transfer. Others have used ontologies to store the knowledge acquired by the application and others have made web-services. Regarding the network, we have the use of LoWPAN, WSN, and WBAN. One of the most used standards was the WBAN, presented in five of the articles. The few standards presented in the articles are not being used even for half of the studies studied. One of the premises of the IoTs is precisely the fact that all things and sensors capture full-time data to be used by the most diverse applications, but if there is no portability between them, we would have only sensor systems and not a true IoTs. In the studied papers, a few standards were used.

In this context, a great possibility of exploration and studies would be to propose standards for the IoTs in health's area. Thus, standard architectures for systems could be proposed, for all research to follow the same path that works. Another idea would be to propose standards for the data captured by the sensors, so any data captured by any sensor of any application could be used by any other application. Even in this context, another possibility could be the proposal for data standards handled by these applications. In this way, the applications would have standards for the data made available to the user, so that the output data of an approach could be used as input to any other approach. Thus, the systems proposed by several future studies would have the capacity to exchange information between them in a natural and intuitive way, to effectively realise a collaborative IoTs, utilising the full potential of technology. 


\subsection{Security and privacy}

Another point to be highlighted after analysing the articles is the issue of security and privacy. Few approaches have been concerned with the safety of patient data. And even fewer have been able to implement adequate systems to address this problem. The security and privacy problem is extremely critical to the IoTs. With data being generated about users, and the objects that belong to them, the amount of information that can be generated is incalculable. In addition, in most cases, this information can be classified as confidential, since users would not like to make it available to third parties. Thus, the need to provide resources to deal with security and privacy of data captured is extremely valuable to the scientific and industrial approaches.

In this context, a possibility of exploration would be to develop systems capable of implementing an efficient and transparent layer of security to the users, that can protect the data of the same of a simple, but safe, way. Currently, one of the technologies that have been gaining a lot of prominences when it comes to data security is blockchain. Blockchain provides an interesting approach to maintaining reliable databases on untrusted networks. Initially, it was used in bitcoin proposed by Nakamoto (2008) as a data structure, becoming a technological solution that provides security in distributed systems (Jin et al., 2017). According to Kuzuno and Karam (2017), blockchain is an electronic records book that keeps a public record of all transactions processed in full transparency without revealing the identity of the transmitter and receiver. In this way, systems that use Blockchain are extremely secure, and can become what is needed to provide adequate security for the IoTs, and to protect user data in that environment.

\subsection{Prediction on healthcare environments}

Most of the studied approaches focus on predicting future patient health conditions. Based on this predictive strategy, applications are able to inform patients, doctors, and family members when bad situations will happen so that preventive measures are taken. In this way, risk situations can be minimised and even avoided depending on the prediction time. However, not only the vital signs are important when we talk about the health of a patient. People are exposed to various situations that may endanger their health. And besides, one of the most common problems when someone seeks hospital care is the precarious situation of emergencies in Brazil.

In this context, one of the possibilities of exploration would be to propose systems capable of predicting external factors that influence the health of patients in hospitals. The goal is capture information from the environment where the patient is inserted, in order to anticipate problems that may affect him. One possibility would be to analyse the resources that a given hospital has and uses to attend to the public, to identify underutilisation or overuse of them and, based on past use, to predict when a given resource will be insufficient to meet the demand. In this way, hospital systems are aware of which features are insufficient, what resources are left over, and what resources are going to become insufficient, can they optimise processes, perhaps by eliminating unnecessary resources, and focusing on acquiring those that really are or will be needed. 


\section{Conclusions}

This study aimed to discuss the main issues regarding the use of data forecast combined with IoT applied to healthcare and identify the concepts of the technology in this area. Differently of others works, our survey do not address only the use of IoT in healthcare field, but also the use of IoT combined with data forecast in order to antecipate and mitigate problems in this area of study. In this context we were able to identify and propose a broad taxonomy for the scope of work, and an updated view of challenges and possibilities for future studies in this area. However, our survey is limited to the use of IoT and data forecast techniques applied to healthcare area. Furthermore, this work just contemplates articles published in electronic scientific libraries, not analysing other types of work, such as technical or industrial approaches.

We expected that the taxonomy proposed in this work can help in the classification of different systems using the IoTs concept combined with data forecast for solving problems in healthcare, serving as baseline of studies for the scientific community in general. We hope that, with this taxonomy, future approaches will be able to identify gaps in healthcare systems, and show ways for future new approaches in the area. We also hope that our research can help hospital IT teams and researchers to propose systems and approaches that can help treat patients in a more automated way with techniques that can predict health problems and prevent them from happening.

As future work, we envision the development of a model for controlling processes in a healthcare environment, using the IoTs paradigm, to record the use of resources by healthcare environment processes, being able to evaluate and predict when the demand for some resource will exceed the capacity of use of the same resource. Therefore, it would be necessary to define which sensors to use and which places they should be allocated, in order to perform data capture for some time. With a good amount of data, data forecast techniques could be applied to identify patterns and predict, with a good degree of confidence, the overuse of some resource. Finally, we expected to implement the system in a real healthcare environment, in order to have real data for a better validation of the proposed model.

\section{References}

Akeju, O., Butakov, S. and Aghili, S. (2018) 'Main factors and good practices for managing BYOD and IoT risks in a K-12 environment', International Journal of Internet of Things and Cyber-Assurance, Vol. 1, No. 1, pp.22-39.

Ali, F., Islam, S.R., Kwak, D., Khan, P., Ullah, N., Jo Yoo, S. and Kwak, K. (2018) 'Type-2 fuzzy ontology - aided recommendation systems for IoT-based healthcare', Computer Communications, Vol. 119, No. 1, pp.138-155.

Azimi, I., Anzanpour, A., Rahmani, A.M., Pahikkala, T., Levorato, M., Liljeberg, P. and Dutt, N. (2017) 'HiCH: hierarchical fog-assisted computing architecture for healthcare IoT', $A C M$ Transactions on Embedded Computing Systems (TECS), Vol. 16, No. 5s, pp.174:1-174:20.

Chen, J., Chen, H., Wu, Z., Hu, D. and Pan, J.Z. (2017) 'Forecasting smog-related health hazard based on social media and physical sensor', Information Systems, Vol. 64, No. Supplement C, pp.281-291.

Darshan, K.R. and Anandakumar, K.R. (2015) 'A comprehensive review on usage of internet of things (iot) in healthcare system', in 2015 International Conference on Emerging Research in Electronics, Computer Science and Technology (ICERECT), IEEE, Mandya, India, pp.132-136. 
EndNote (2017) [online] https://endnote.com/ (accessed 16 November 2017).

Farahani, B., Firouzi, F., Chang, V., Badaroglu, M., Constant, N. and Mankodiya, K. (2018) 'Towards fog-driven IoT e-health: promises and challenges of IoT in medicine and healthcare', Future Generation Computer Systems, Vol. 78, No. Part 2, pp.659-676.

Jin, T., Zhang, X., Liu, Y. and Lei, K. (2017) 'BlockNDN: a bitcoin blockchain decentralized system over named data networking', in 2017 Ninth International Conference on Ubiquitous and Future Networks (ICUFN), IEEE, Milan, Italy, pp.75-80.

Jouini, R., Lemlouma, T., Maalaoui, K. and Saidane, L.A. (2016) 'Employing grey model forecasting $\operatorname{GM}(1,1)$ to historical medical sensor data towards system preventive in smart home e-health for elderly person', in 2016 International Wireless Communications and Mobile Computing Conference (IWCMC), IEEE, pp.1086-1091.

Kan, C., Chen, Y., Leonelli, F. and Yang, H. (2015) 'Mobile sensing and network analytics for realizing smart automated systems towards health internet of things', in 2015 IEEE International Conference on Automation Science and Engineering (CASE), IEEE, pp.1072-1077.

Kang, J.J., Adibi, S., Larkin, H. and Luan, T. (2015) 'Predictive data mining for converged internet of things: a mobile Health perspective', in 2015 International Telecommunication Networks and Applications Conference (ITNAC), IEEE, pp.5-10.

Kuzuno, H. and Karam, C. (2017) 'Blockchain explorer: an analytical process and investigation environment for bitcoin', in 2017 APWG Symposium on Electronic Crime Research (eCrime), IEEE, Scottsdale, AZ, USA, pp.9-16.

Kwon, O.Y., Shin, S.H., Shin, S.J. and Kim, W.S. (2010) 'Design of u-health system with the use of smart phone and sensor network', in 2010 Proceedings of the 5th International Conference on Ubiquitous Information Technologies and Applications, IEEE, pp.1-6.

Meharouech, A., Elias, J. and Mehaoua, A. (2015) 'Future body-to-body networks for ubiquitous healthcare: a survey, taxonomy and challenges', in 2015 2nd International Symposium on Future Information and Communication Technologies for Ubiquitous HealthCare (Ubi-HealthTech), IEEE, Beijing, China, pp.1-6.

Nakamoto, S. (2008) Bitcoin: A Peer-to-Peer Electronic Cash System [online] https://bitcoin.org/ bitcoin.pdf (accessed 30 May 2018).

Orimaye, S.O., Leong, F.C., Lee, C.H. and Ng, E.C.H. (2015) 'Predicting proximity with ambient mobile sensors for non-invasive health diagnostics', in 2015 IEEE 12th Malaysia International Conference on Communications (MICC), IEEE, pp.6-11.

Pinto, S., Cabral, J. and Gomes, T. (2017) 'We-care: an IoT-based health care system for elderly people', in 2017 IEEE International Conference on Industrial Technology (ICIT), IEEE, Toronto, ON, Canada, pp.1378-1383.

Rahmani, A.M., Gia, T.N., Negash, B., Anzanpour, A., Azimi, I., Jiang, M. and Liljeberg, P. (2018) 'Exploiting smart e-health gateways at the edge of healthcare internet-of-things: a fog computing approach', Future Generation Computer Systems, Vol. 78, No. Part 2, pp.641-658.

Robben, S., Englebienne, G. and Kröse, B. (2017) 'Delta features from ambient sensor data are good predictors of change in functional health', IEEE Journal of Biomedical and Health Informatics, Vol. 21, No. 4, pp.986-993.

Roehrs, A., da Costa, C.A., da Rosa Righi, R. and de Oliveira, K.S.F. (2017) 'Personal health records: a systematic literature review', Journal of Medical Internet Research, Vol. 19, No. 1, p.e13.

Sarhan, Q.I. (2018) 'Internet of things: a survey of challenges and issues', International Journal of Internet of Things and Cyber-Assurance, Vol. 1, No. 1, pp.40-75.

Singh, K.J. and Kapoor, D.S. (2017) 'Create your own internet of things: a survey of IoT platforms', IEEE Consumer Electronics Magazine, Vol. 6, No. 2, pp.57-68. 
Spanakis, E.G., Kafetzopoulos, D., Yang, P., Marias, K., Deng, Z., Tsiknakis, M., Sakkalis, V. and Dong, F. (2014) 'Myhealthavatar: personalized and empowerment health services through internet of things technologies', in 2014 4th International Conference on Wireless Mobile Communication and Healthcare - Transforming Healthcare Through Innovations in Mobile and Wireless Technologies (MOBIHEALTH), IEEE, pp.331-334.

Vargheese, R. and Viniotis, Y. (2014) 'Influencing data availability in IoT enabled cloud based e-health in a 30 day readmission context', in 10th IEEE International Conference on Collaborative Computing: Networking, Applications and Worksharing, IEEE, pp.475-480.

Zamanifar, A., Nazemi, E. and Vahidi-Asl, M. (2017) 'DMP-IoT: a distributed movement prediction scheme for IOT health-care applications', Computers \& Electrical Engineering, Vol. 58, No. Supplement C, pp.310-326. 